\title{
Serum IgE and IgG Reactivity to Aspergillus Recombinant Antigens in Patients with Cystic Fibrosis with Aspergillus sensitisation, ABPA and Aspergillus bronchitis
}

Nada S. Alghamdi (University of Leeds, UK), Richard Barton (Leeds Teaching Hospitals Trust, UK), Mark Wilcox (Leeds Teaching Hospital, Leeds, UK), Daniel Peckham (Faculty of Medicine and Health, University of Leeds, Leeds, UK).

The diagnosis of aspergillosis in cystic fibrosis (CF) remains a challenge due to overlapping features of both diseases. This is further complicated by inconsistent antibody reactivity to the currently used crude antigen, which has led a more focused evaluation of the efficacy of IgE response to a number of pure Aspergillus fumigatus (Af) recombinant proteins in order to effect a diagnosis. In this study, we dissected the $\lg E$ and $\lg G$ responses to multiple Af recombinant antigens in CF patients with different Aspergillus diseases.

Methods: Serum IgE and IgG antibodies were measured in 12 CF patients with Allergic Bronchopulmonary Aspergillosis (ABPA), 12 with Aspergillus sensitization, and 12 with Aspergillus bronchitis against recombinant antigens Asp f1, f2, $\mathrm{f} 3$, f4, and f6.

ABPA criteria: elevated Af IgE levels (>0.35 kUA/l), raised total lgE levels $(>1000 \mathrm{IU} / \mathrm{ml})$, and at least two of the following: presence of Af IgG (>90 mgA/l), radiological findings compatible with ABPA, or eosinophilia (>0.5 x 109/I).

Aspergillus Bronchitis (AB) criteria: elevated Aspergillus IgG (>90 mgA/l), and positive sputum culture for Aspergillus species.

Aspergillus sensitization (AS) criteria: elevated Af IgE levels (>0.35 kUA/l).

Results: IgE antibodies in the ABPA group significantly reacted to Asp f1, 2 and 4. There was generally low $\lg E$ response to these antigens in AS patients. Aspergillus bronchitis samples showed higher lgG positivity to Asp f1 and Asp f2 compared with ABPA. (See Figure)
Sera from ABPA patients with positive culture showed a higher median lgG level to Asp f1 compared with those with negative culture.

Conclusion: Whilst AS patient samples could be differentiated from ABPA samples based purely on total lgE levels, the use of multiple recombinant antigens may improve the diagnostic accuracy in CF complicated with ABPA or Aspergillus bronchitis. Specifically, IgG Aspf1 reactivity may relate to the presence of actively growing Aspergillus spp. which might be a useful marker for guiding antifungal therapy in both $A B$ and subsets of ABPA patients.

\begin{tabular}{|c|c|c|c|c|c|c|}
\hline $\begin{array}{l}\text { Patient } \\
\text { groups }\end{array}$ & $\begin{array}{l}\text { Sex } \\
f / m\end{array}$ & $\begin{array}{c}\text { Age } \\
\text { (median, } \\
\text { range) }\end{array}$ & $\begin{array}{l}\text { Asp } \\
\lg G \\
\mathrm{mg} /\left.\right|^{\star}\end{array}$ & $\begin{array}{l}\text { Asp } \\
\lg E \\
|U /|^{\star}\end{array}$ & $\begin{array}{l}\text { Total } \\
\text { IgE } \\
\text { IU//* }\end{array}$ & $\begin{array}{c}\text { Culture } \\
\text { positive } \\
\star \star\end{array}$ \\
\hline ABPA & $4 / 8$ & $16,3-23$ & 132 & 26 & 2210 & $6 / 10$ \\
\hline$A B$ & $7 / 5$ & $25,5-36$ & 130 & $<0.35$ & 11 & $12 / 12$ \\
\hline AS & $3 / 9$ & $26,13-52$ & 48 & 5 & 130 & $3 / 12$ \\
\hline
\end{tabular}

Table: Characteristics of patients studied. (ABPA: Allergic Bronchopulmonary Aspergillosis, AS: Aspergillus Sensitization, AB: Aspergillus Bronchitis).

* Median results

** Positive culture for Aspergillus fumigatus.
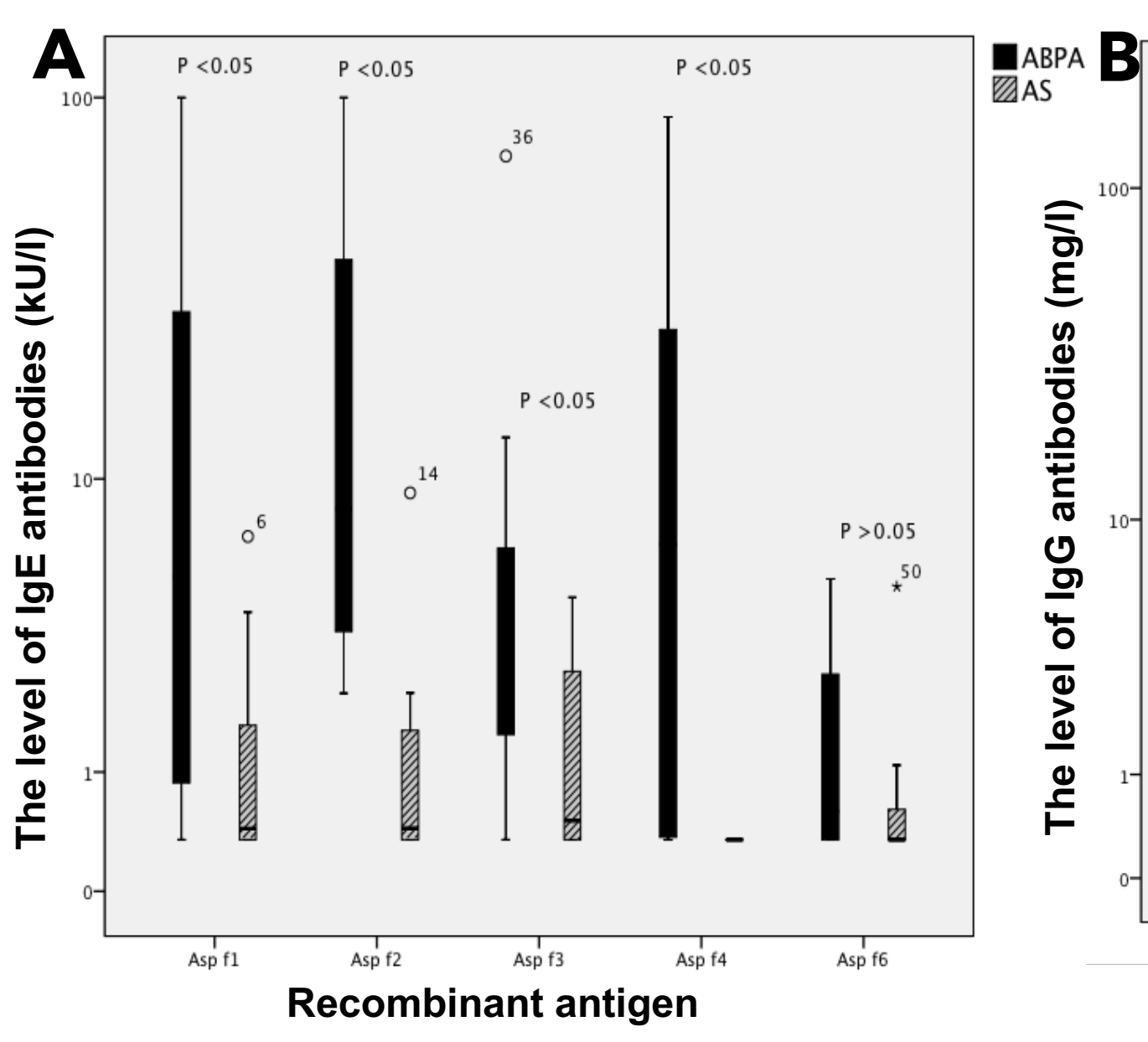

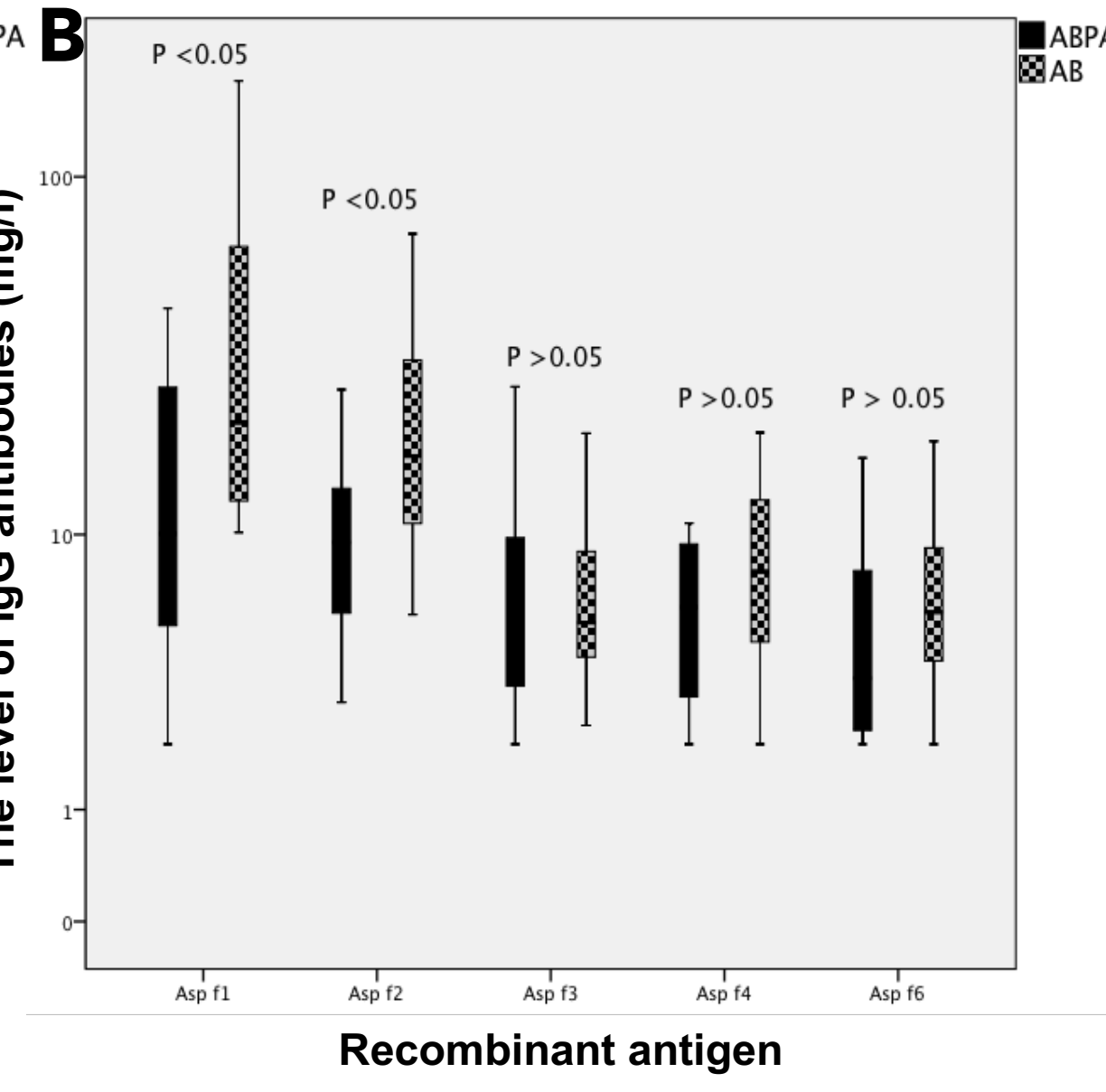

Copyright @ 2017 Nada Alghamdi, Richard Barton, Mark Wilcox, Daniel Peckham
Figure: Box plots of the median and interquartile range of: (A) Serum IgE reactivity to recombinant antigens Asp $f 1,2,3,4$, and 6 in ABPA and AS patients. (B) Serum IgG reactivity pattern to recombinant antigens Asp

$f 1,2,3,4$, and 6 in ABPA and $A B$ patients.

o, * outliers, $\square$ ABPA, 근 